\title{
СРАВНЕНИЕ РЕЗУЛЬТАТОВ ИОНОМЕТРИЧЕСКОГО И ИОНОХРОМАТОГРАФИЧЕСКОГО ОПРЕДЕЛЕНИЙ НИТРАТ- И ХЛОРИДИОНОВ В ОВОЩАХ
}

\author{
(Представил О. Эйзен)
}

Качество и сохранность овощей зависят от наличия в них нитрат- и хлоридионов. Высокое содержание хлоридионов снижает вкусовые качества и сохранность овощей. Нитратионы в продуктах питания представляют опасность для здоровья человека, так как являются потенциальными источниками канцерогенных веществ (нитрозаминов). Поэтому Министерство здравоохранения СССР утвердило предельно допустимые содержания нитратионов в овощах [']. Соответствующий контроль требует массовых количественных определений нитрат- и хлоридионов в овощах. Наряду с колориметрическими методами наиболее перспективны два относительно новых метода анализа - ионометрия с применением ионоселективных электродов (ИСЭ) и ионная хроматография (ИХ). Цель настоящей работы - сравнение результатов определения нитрат- и хлоридионов этими методами.

\section{Экспериментальная часть}

Получение водной вытяжки, содержащей нитрат- и хлоридионы. Среднюю пробу овощей измельчали до $1-2$ мм и брали навеску от 5 до 25 г в зависимости от предполагаемого содержания в материале $\mathrm{NO}_{3}^{-}$и $\mathrm{Cl}^{-}$. Для получения вытяжки к навеске овощей добавляли водный раствор электролитов: либо $0,03 \mathrm{M} \mathrm{Al}_{2}\left(\mathrm{SO}_{4}\right)_{3}+0,06 \mathrm{M} \mathrm{H}_{3} \mathrm{BO}_{3}$ (для ионометрических определений $\mathrm{NO}_{3}^{-}$и $\mathrm{Cl}^{-}$), либо $0,024 \mathrm{M} \mathrm{Na}_{2} \mathrm{CO}_{3}$ (для ионохроматографических определений $\mathrm{NO}_{3}^{-}$и $\mathrm{Cl}^{-}$). В том и другом случае раствор электролитов с навеской овощей выдерживали 2 ч на кипящей водяной бане, раствор охлаждали, доводили до $100 \mathrm{~cm}^{3}$ и фильтровали через вату для удаления твердой фазы. Профильтрованный раствор центрифугировали 15 мин при 3000 об/мин. В центрифугате определяли содержание $\mathrm{NO}_{3}^{-}$и $\mathrm{Cl}^{-}$.

Рис. 1. Схема ионного хроматографа: 1 - емкость с элюентом, 2 - насос, 3 - дозатор, 4 - разделяющая колонка, 5 - компенсационная колонка, 6 - детектор.

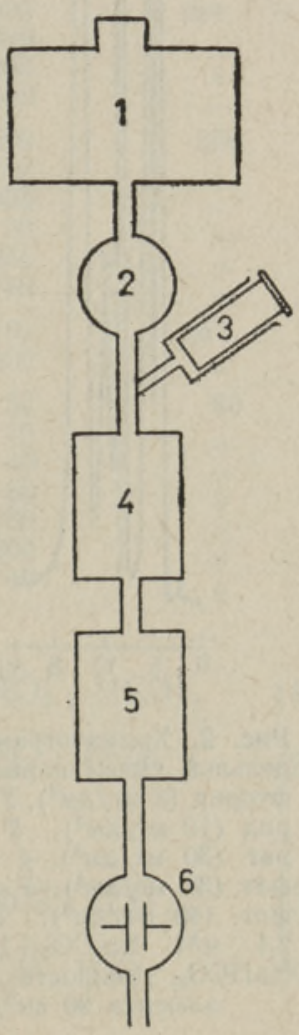


Ионометрическая аппаратура и методика определения $\mathrm{NO}_{3}^{-}$и $\mathrm{Cl}^{-}$. Ионометрическая установка состояла из рН-метра OP-208 (фирмы «Radelkis», Венгрия), электрода сравнения K701 (фирмы «Radiometer», Дания) и ионоселективных электродов $Э \mathrm{M}-\mathrm{NO}_{3}-01$ для нитратионов (производства $\mathrm{CCCP)} \mathrm{и} \mathrm{F} 1012 \mathrm{Cl}$ для хлоридионов (фирмы «Radiometer»). Концентрацию нитратионов определяли методом добавок $\left.{ }^{2}\right]$, концентрацию хлоридионов - по калибровочной кривой. Добавленные и калибровочные растворы выравнивали с исследуемым по концентрациям $\mathrm{Al}_{2}\left(\mathrm{SO}_{4}\right)_{3}$ и $\mathrm{H}_{3} \mathrm{BO}_{3}$.

Аппаратура и методика определения $\mathrm{NO}_{3}^{-}$и $\mathrm{Cl}^{-}$методом ионной хроматографии. Использовали ионный хроматограф, построенный в химической лаборатории Тартуской санэпидстанции (рис. 1). Разделяющую колонку (длина 30 см, диаметр 3 мм) заполняли сорбентом, синтезированным в Институте химии АН ЭССР, а компенсационную - катионитом КУ-2 (фракция 70-100 мкм). Объем вводимого для анализа раство$\mathrm{pa}-0,100 \mathrm{~cm}^{3}$. Минимально определяемые концентрации $\mathrm{NO}_{3}^{-}$и $\mathrm{Cl}^{-}-2$

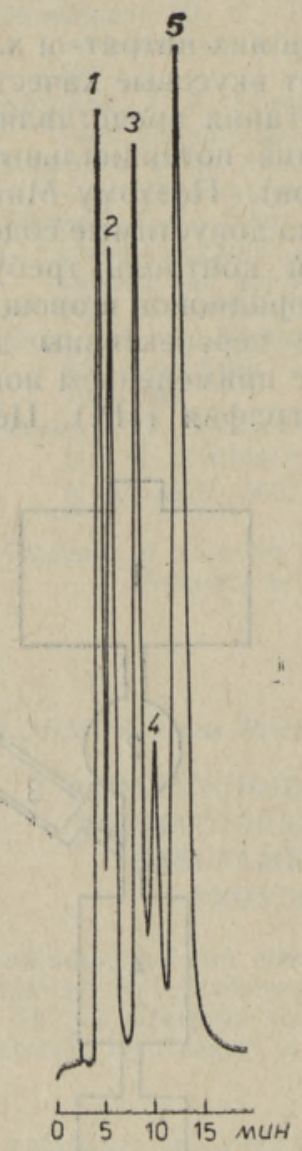

Рис. 2. Хроматограмма модельной, смеси ионов: 1 фторид (8 мг/дм $\left.{ }^{3}\right), 2$ - хлорид $\left(10 \mathrm{мг} /\right.$ дм $\left.^{3}\right), 3-$ ннтрат $\left(30\right.$ мг/дм $\left.{ }^{3}\right), 4-$ фосфат $\left(30 \mathrm{mr} /\right.$ дм $\left.^{3}\right), 5$ - сульфат $\left(40 \mathrm{mr} /\right.$ дм $\left.^{3}\right)$. Элюент: $2,4 \quad \mathrm{MM} \quad \mathrm{Na}_{2} \mathrm{CO}_{3}+1,5 \quad$ мM $\mathrm{NaHCO}_{3}$, скорость подачи элюента $90 \mathrm{~cm}^{3} /$ ч. a

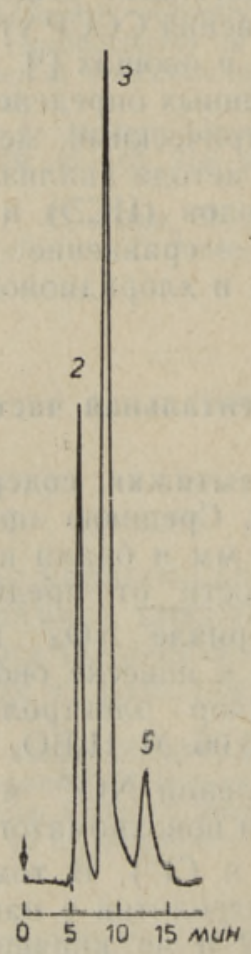

Рис. 3. Хроматограммы экстрактов: $a-$ салатного (2-хлорнд 6,1 мг/дм ${ }^{3}, 3$ - нит. рат $39 \mathrm{мr} /$ дм$^{3}, 5-$ сульфат +оксалат); 6 - огуречного ( 1 - фторид + неидентифицированный аннон, 2 - хлорнд $6,2 \mathrm{мг} /$ дм $^{3}$, 3 - нитрат $14 \mathrm{мг} /$ дм $^{3}, 4$ - фосфат + нендентифицированный анион, 5 - сульфат + оксалат). Состав элюента и скорость его подачи см. в подписи к рис. 2. 
и 0,1 мкг $/$ см $^{3}$ соответственно. Хроматограмму модельной смеси (рис. 2) использовали для идентификации и калибровки. Содержание $\mathrm{NO}_{3}^{-}$и Cl- в вытяжках овощей определяли по калибровочным графикам в координатах высота пика-концентрация иона (рис. 3).

\section{Результаты и их обсуждение}

Результаты определений нитрат- и хлоридионов (таблица) обрабатывали методом регрессионного анализа по уравнению $y=a+b x$, где $x-$ безошибочный аргумент. Для тех и других ионов получили по два регрессионных уравнения: типа А, когда в качестве $x$ использовали результаты ионометрических определений, и типа Б, когда в качестве $x$ использовали результаты ионохроматографических определений. Такой подход обусловлен тем, что практически ни один из рассматриваемых методов анализа нельзя считать безошибочным и все расхождения приписывать к ошибкам одного из них.

Содержание $\mathrm{Cl}^{-}$и $\mathrm{NO}_{3}^{-}$в овощах по данным ионной хроматографии (ИХ) и ионометрии с использованием ионоселективных электродов (ИСЭ)

\begin{tabular}{|c|c|c|c|c|c|}
\hline \multirow{2}{*}{$\begin{array}{l}\text { Объект } \\
\text { анализа }\end{array}$} & \multicolumn{2}{|c|}{$\mathrm{Cl}^{-}, \mathrm{Mr} / \mathrm{kr}$} & \multicolumn{3}{|c|}{$\mathrm{NO}_{3}^{-}, \mathrm{Mr} / \mathrm{Kr}$} \\
\hline & их & ИСЭ & их & ИСЭ & норма ['] \\
\hline $\begin{array}{c}\text { Свекла } \\
\text { столовая } \\
" \\
"\end{array}$ & $\begin{array}{r}1040 \\
1280 \\
820 \\
2160\end{array}$ & $\begin{array}{r}1140 \\
1100 \\
800 \\
2060\end{array}$ & $\begin{array}{r}720 \\
1360 \\
1000 \\
1520\end{array}$ & $\begin{array}{r}760 \\
1290 \\
1030 \\
1500\end{array}$ & $\begin{array}{c}1400 \\
" \\
"\end{array}$ \\
\hline $\begin{array}{c}\text { Салат } \\
\text { ” } \\
\text { " }\end{array}$ & $\begin{array}{l}1240 \\
3400 \\
2300 \\
1800\end{array}$ & $\begin{array}{l}1620 \\
2700 \\
2800 \\
2600\end{array}$ & $\begin{array}{l}7800 \\
9800 \\
5200 \\
9200\end{array}$ & $\begin{array}{l}6600 \\
7300 \\
4800 \\
7500\end{array}$ & $\begin{array}{r}\text { нет } \\
", \\
",\end{array}$ \\
\hline $\begin{array}{c}\text { Огурцы } \\
\text { " } \\
" \\
" \\
"\end{array}$ & $\begin{array}{l}290 \\
250 \\
200 \\
360 \\
500 \\
140\end{array}$ & $\begin{array}{l}340 \\
290 \\
300 \\
440 \\
600 \\
210\end{array}$ & $\begin{array}{r}650 \\
420 \\
320 \\
80 \\
660 \\
700\end{array}$ & $\begin{array}{r}620 \\
410 \\
350 \\
50 \\
650 \\
780\end{array}$ & $\begin{array}{l}300 \\
" \\
" \\
" \\
"\end{array}$ \\
\hline $\begin{array}{r}\text { Редис } \\
\text {, }\end{array}$ & $\begin{array}{l}260 \\
520\end{array}$ & $\begin{array}{l}500 \\
780\end{array}$ & $\begin{array}{l}3400 \\
4800\end{array}$ & $\begin{array}{l}4100 \\
5000\end{array}$ & $\begin{array}{r}\text { нет } \\
\text { ", }\end{array}$ \\
\hline $\begin{array}{c}\text { Қартофель } \\
\text { ” } \\
, \\
" \\
" \\
,\end{array}$ & $\begin{array}{l}580 \\
360 \\
720 \\
800 \\
720 \\
700 \\
990\end{array}$ & $\begin{array}{l}700 \\
500 \\
820 \\
650 \\
790 \\
860 \\
820\end{array}$ & $\begin{array}{r}88 \\
90 \\
49 \\
90 \\
210 \\
180 \\
70\end{array}$ & $\begin{array}{r}52 \\
70 \\
40 \\
66 \\
180 \\
200 \\
40\end{array}$ & $\begin{array}{l}80 \\
", \\
", \\
", \\
",\end{array}$ \\
\hline
\end{tabular}

Уравнения типа A:

$$
\begin{gathered}
C\left(\mathrm{NO}_{3}^{-}, \text {ИХ }\right)=(-144 \pm 263)+(1,19 \pm 0,08) \cdot C\left(\mathrm{NO}_{3}^{-}, \text {ИСЭ }\right), \\
s=484, s / \Delta y=0,053, r=0,988 ; \\
C\left(\mathrm{Cl}^{-}, \text {ИХ }\right)=(-45 \pm 203)+(0,96 \pm 0,16) \cdot C\left(\mathrm{Cl}^{-}, \text {ИСЭ }\right), \\
s=282, s / \Delta y=0,087, r=0,940 .
\end{gathered}
$$

Уравнения типа Б:

$$
\begin{aligned}
\mathrm{C}\left(\mathrm{NO}_{3}^{-}, \text {иСЭ }\right) & =(162 \pm 212)+(0,82 \pm 0,06) \cdot \mathrm{C}\left(\mathrm{NO}_{3}^{-}, \text {иХ }\right), \\
s & =401, s / \Delta y=0,054, r=0,988 \\
\mathrm{C}(\mathrm{Cl}-, \text { иСЭ }) & =(161 \pm 186)+(0,92 \pm 0,15) \cdot \mathrm{C}\left(\mathrm{Cl}^{-}, \text {иХ }\right), \\
s & =276, s / \Delta y=0,107, r=0,940 .
\end{aligned}
$$


В уравнениях (1)-(4) доверительные интервалы регрессионных коэффициентов вычислены на уровне $P=0,95\left[^{3}\right]$. Приведены соответствующие среднеквадратичные отклонения [3], вычисленные по формуле

$$
s=\left(\sum_{i=1}^{i=n}\left(y_{i}^{\text {эксп }}-y_{i}^{\text {pacu }}\right)^{2} /(n-2)\right)^{0,5}
$$

где $n-$ число точек, и отношение $s / \Delta y\left[{ }^{4}\right]$, где $\Delta y-$ диапазон изменения $y$ (разность между максимальным и минимальным значениями $y$ ).

На основе полученных значений $s / \Delta y$ и $r$ можно сделать вывод, что результаты ионной хроматографии и ионометрических определений исследованных ионов удовлетворительно согласуются между собой. При этом свободный член в уравнениях (1)-(4) несуществен на уровне доверительной вероятности $P=0,95$ и может быть опущен (принят равным нулю). Следовательно, регрессионную зависимость между ионохроматографическими и ионометрическими определениями нитрат- и хлоридионов можно искать по уравнению $y=b x$, что приводит к следующим соотношениям.

Уравнения типа A:

$$
\begin{aligned}
& \mathrm{C}\left(\mathrm{NO}_{3}^{-}, \mathrm{XX}\right)=(1,16 \pm 0,07) \cdot \mathrm{C}\left(\mathrm{NO}_{3}^{-}, \mathrm{HC}\right) \text {, } \\
& s=487, s / \Delta y=0,053 \text {; } \\
& \mathrm{C}\left(\mathrm{Cl}^{-}, \text {ИХ }\right)=(0,93 \pm 0,09) \cdot \mathrm{C}\left(\mathrm{Cl}^{-}, \text {ИСЭ }\right) \text {, } \\
& s=277, s / \Delta y=0,085 \text {. }
\end{aligned}
$$

Уравнения типа Б:

$$
\begin{aligned}
& \mathrm{C}\left(\mathrm{NO}_{3}^{-}, \text {ИСЭ }\right)=(0,84 \pm 0,05) \cdot \mathrm{C}\left(\mathrm{NO}_{3}^{-}, \mathrm{KX}\right) \text {, } \\
& s=415, s / \Delta y=0,056 \\
& \mathrm{C}\left(\mathrm{Cl}^{-}, \text {ИС }\right)=(1,02 \pm 0,10) \cdot \mathrm{C}\left(\mathrm{Cl}^{-}, \mathrm{KX}\right) \text {, } \\
& s=290, s / \Delta y=0,112 \text {, }
\end{aligned}
$$

где

$$
S=\left(\sum_{i=1}^{i=n}\left(y_{i}^{\text {эксп }}-y_{i}^{\text {pacq }}\right)^{2} /(n-1)\right)^{0,5}
$$

Из уравнений (6) и (8) видно, что ионометрические определения нитратионов дают заниженные в среднем на $16 \%$ результаты по сравнению с полученными методом ионной хроматографии. Следует отметить, что ионометрические измерения проводятся на фоне других электролитов, отчего результаты этих измерений менее надежны, чем определенные ионохроматографически. Уравнения (7) и (9) свидетельствуют о том, что в случае определения хлоридионов систематических расхождений между результатами, полученными обоими методами, нет; соответствующий регрессионный коэффициент может быть приравнен единице.

Среднеквадратичные отклонения (10), вычисленные из регрессий (6) - (9), составляют 5-11\% от диапазона изменения функции $y$, в направлении которой минимизировалась сумма квадратов отклонений (см. величины $s / \Delta y)$.

Из представленных в таблице данных можно вычислить среднеквадратичное относительное расхождение результатов, полученных методами ионометрии и ионной хроматографии:

$$
s_{\text {oтH }}=\left(\sum_{i=1}^{i=n} E_{i}^{2} /(n-1)\right)^{0,5}
$$

где

$$
E_{i}=\frac{C_{(\mathrm{IIx})}-C_{(\mathrm{IIC3)}}}{0,5 \cdot\left(C_{(\mathrm{ux})}+C_{(\mathrm{He}) \mathrm{s})}\right.} .
$$


Величина $s_{\text {отн }}$ для нитрат- и хлоридионов равна 0,237 и 0,271 соответственно, т. е. среднее расхождение между результатами, полученными обоими методами, составляет $24-27 \%$ от измеряемой величины.

\section{Выводы}

1. Разработаны ионохроматографический и ионометрический методы количественного определения нитрат- и хлоридионов в овощах.

2. Проведено сравнение результатов, полученных обоими методами при анализе одних и тех же объектов.

3. Показано, что оба метода анализа дают согласующиеся результаты, однако результаты определения нитратионов методом ионометрии в среднем на $16 \%$ меньше полученных методом ионной хроматографии.

4. Среднее расхождение обоих методов составляет $24-27 \%$ (отн.).

\section{Л И Т Е Р А Т У Р А}

1. Допустимое содержание нитратов в растительных пищевых продуктах и рекомендации по санитарно-гигиеническому контролю за их содержанием. М., 1984.

2. Mascini, M. Uses of known addition, Gran's plots and the related methods with ion-selective electrodes. - In: Ion-Selective Rev., 2. Great Britain, 1980, 17-71.

3. Доерффель К. Статистика в аналитической химии. М., 1969, 178-185.

4. Koppel, I. A., Palm, V. A. In: Advances in Linear Free Energy Relationships. London, 1972, Chapter 5.

Тартуская городская санэпидстанция

Поступила в редакцию

Институт химии

$28 /$ II 1985

Академии наук Әстонской ССР

J. PENTSUK, U. HALDNA, K. ILMOJA, M. IVASK

\section{AEDVILJADES LEIDUVATE NITRAAT- JA KLORIIDIOONIDE IOONKROMATOGRAAFILISE NING IONOMEETRILISE MAARAMISE TULEMUSTE VORDLEMINE}

Nitraat- ja kloriidioonide määramiseks aedviljades on välja töötatud ioonkromatograafiline ja ionomeetriline metoodika. Nende meetoditega saadud analüüsitulemused on omavahel rahuldavas kooskōlas: suhteline keskmine tulemuste erinevus meetodite vahel on $24-27 \%$. Leiti, et ionomeetriline metoodika annab keskmiselt $16 \%$ madalamad nitraatiooni sisaldused aedviljas kui ioonkromatograafiline metoodika.

\section{J. PENCHUK, O. HALDNA, K. ILMOJA, M. IVASK}

\section{COMPARISON OF RESULTS OBTAINED BY ION CHROMATOGRAPHY AND ION-SELECTIVE ELECTRODES ON THE DETERMINATION OF NITRATE AND CHLORIDE IONS IN VEGETABLES}

Ion-chromatographic and potentiometric methods using ion-selective electrodes have been developed for nitrate and chloride determination in vegetables. The agreement between the results obtained by the two methods is satisfactory, the relative mean difference between those results being $24-27 \%$. It has been found that the nitrate concentration obtained by the use of ion-selective electrodes is on the average by $16 \%$ lower than that obtained by ion chromatography. 